\title{
Search for Baryonic Resonances Decaying to $\Xi \pi$ in Deep-Inelastic Scattering at HERA
}

\author{
Marc Del Degan \\ ETH Zürich - Institut für Teilchenphysik \\ Schafmattstr. 20 - Switzerland \\ on behalf of the $\mathrm{H} 1$ Collaboration
}

\begin{abstract}
A search for narrow baryonic resonances decaying into $\Xi^{-} \pi^{-}$or $\Xi^{-} \pi^{+}$and their antiparticles is carried out with the $\mathrm{H} 1$ detector using deep inelastic scattering events at HERA in the range of negative photon four-momentum transfer squared $2<Q^{2}<$ $100 \mathrm{GeV}^{2}$. No signal for a new baryonic state in the mass range $1600-2300 \mathrm{MeV}$ is observed in either the doubly charged or the neutral decay channels. The known baryon $\Xi(1530)^{0}$ is observed through its decay mode into $\Xi^{-} \pi^{+}$. Mass/dependent upper limits are given on the ratio of the production rates of new baryonic states, such as the hypothetical pentaquark states $\Xi_{5 q}^{--}$or $\Xi_{5 q}^{0}$, relative to the $\Xi(1530)^{0}$ baryon state.
\end{abstract}

\section{Introduction}

Various theoretical approaches $[2,3,4]$ based on Quantum Chromodynamics predict the existence of exotic baryonic states composed of four valence quarks and an anti-quark, commonly known as "pentaquarks". Such states are expected to form a flavour anti-decuplet and are not explicitly forbidden within the Standard Model.

Several experiments have reported evidence for a narrow resonance with a mass around $1540 \mathrm{MeV}$ decaying into $n K^{+}$and $p K_{S}^{0}$ final states [5]. Such a state could be interpreted as an exotic strange pentaquark with a minimal quark content of $u u d d \bar{s}$, lying in the apex of the spin $1 / 2$ (or $3 / 2$ ) anti-decuplet. On the other hand, a number of other experiments [5], including the H1 experiment [6], have reported non-observation of the same state.

Searches for other members of this anti-decuplet are of interest, in particular searches for the doubly strange $\Xi_{5 q}^{--}(d d s s \bar{u})$ and $\Xi_{5 q}^{+}(u u s s \bar{d})$ states, which are also manifestly exotic. The NA49 collaboration [7] at the SPS reported the observation of two baryonic resonances in fixed targed proton-proton collisions at the centre of mass energy $\sqrt{s}=17.2 \mathrm{GeV}$, with masses of $1862 \pm 2 \mathrm{MeV}$ and $1864 \pm 5 \mathrm{MeV}$, and with widths below the mass resolution of $18 \mathrm{MeV}$. These states can be interpreted as the $\Xi_{5 q}^{--}\left(S=-2, I_{3}=-3 / 2\right)$ and the $\Xi_{5 q}^{0}\left(S=-2, I_{3}=+1 / 2\right)$ members of the isospin $3 / 2$ quartet $\Xi_{3 / 2}$ in the anti-decuplet. These findings have not been confirmed by several other experiments (see for example [8]).

The search presented here is performed using data taken with the H1 detector at HERA. The $\Xi^{-}$particles ${ }^{\text {a }}$ are identified through their decay into $\Lambda \pi^{-}$. The established baryon $\Xi(1530)^{0}[9]$ is observed through its decay mode $\Xi(1530)^{0} \rightarrow \Xi^{-} \pi^{+}$.

\section{Selection of DIS Events}

The data analysed corresponding to an integrated luminosity of $\mathcal{L}=101 \mathrm{pb}^{-1}$ and are taken in the years 1996/1997 and 1999/2000. During this time HERA collided electrons ${ }^{b}$

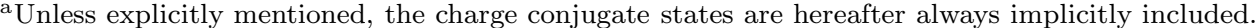

bHerein, the term "electron" is used generically to refer to both electrons and positrons.
} 
at an energy of $27.6 \mathrm{GeV}$ with protons at $820 \mathrm{GeV}\left(1996 / 1997,24.8 \mathrm{pb}^{-1}\right)$ and $920 \mathrm{GeV}$ $\left(1999 / 2000,75.7 \mathrm{pb}^{-1}\right)$.

The negative four momentum transfer squared $Q^{2}$ of the exchanged virtual photon and the inelasticity $y$ as reconstructed from the scattered electron are restricted to the ranges $2<Q^{2}<100 \mathrm{GeV}^{2}$ and $0.05<y<0.7$.

\section{Mass Spectra}

The hypothetical doubly charged $X^{--}$and the neutral $X^{0}$ baryon states are identified by complete reconstruction of their respective decay chains through $\Xi^{-}$and $\Lambda$ baryons into pions and protons, according to

$$
\begin{aligned}
X^{--} & \rightarrow \quad \Xi^{-} \pi^{-} \rightarrow\left[\Lambda \pi^{-}\right] \pi^{-} \rightarrow\left[\left(p \pi^{-}\right) \pi^{-}\right] \pi^{-} \\
X^{0} & \rightarrow \quad \Xi^{-} \pi^{+} \rightarrow\left[\Lambda \pi^{-}\right] \pi^{+} \rightarrow\left[\left(p \pi^{-}\right) \pi^{-}\right] \pi^{+} .
\end{aligned}
$$

In the first step, $\Lambda$ baryons are identified by their charged decay mode, $\Lambda \rightarrow p \pi^{-}$, using pairs of oppositely charged tracks. The track with the higher momentum is assigned the proton mass. The particles are fitted to a common vertex [10] and $\Lambda$ candidates are retained by applying weak kinematic selection criteria. In the second step, $\Xi^{-}$candidates are formed by fitting each of the $\Lambda$ candidates with a negatively charged track assumed to be a pion to a common vertex and applying additional selection criteria. The invariant mass spectra $M\left(\Lambda \pi^{-}\right)$ and $M\left(\bar{\Lambda} \pi^{+}\right)$of all selected $\Xi^{-}$ candidates are shown in figure 1 . In the last step, $X^{--/ 0}$ baryon candidates are formed by combining each $\Xi^{-}$candidate with one additional track, assumed to be a pion and originating at the primary vertex.

The resulting mass spectra for the $X^{0}$ and the $X^{--}$are shown in the upper part of figure 2. In the neutral spectra the signal of the well known $\Xi(1530)^{0}$ state is observed.

A simultaneous fit of the $X^{0}$
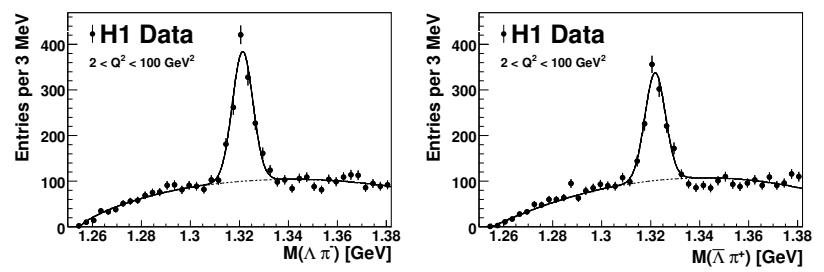

Figure 1: The invariant mass spectra for a) $\Lambda \pi^{-}$and b) $\bar{\Lambda} \pi^{+}$particle combinations. The solid lines show the result of a fit to the data using a Gaussian function for the $\Xi^{-}$signal and a background function as defined in equation 3 (with $m_{\Xi}$ replaced accordingly by $m_{\Lambda}$ ), while the dashed lines indicate the background function only. and the $X^{--}$mass spectra is performed using a function $F$, that contains a gaussian $G$ for the signal $\Xi(1530)^{0}$ baryon and a function $B$ for the background shape, according to

$$
F=G+\left(1+P_{0}\right) B ; \quad B(M)=P_{1}\left(M-m_{\Xi}-m_{\pi}\right)^{P_{2}} \times\left(1+P_{3} M+P_{4} M^{2}\right) .
$$

Here, $M$ denotes the $\Xi \pi$ invariant mass and $m_{\Xi}$ and $m_{\pi}$ the masses of the $\Xi$ and the $\pi$, respectively. The normalisation, the central value and the width of the Gaussian function $G$ as well as the parameters $P_{i}$ are left free in the fit. $P_{0}$ represents the relative normalisation 
of the neutral to the doubly charged combinations and is set to zero for the neutral combinations. The fit yields a total of $163 \pm 24$ (stat.) $\Xi(1530)^{0}$ baryons. The reconstructed mass of $1532 \pm 2$ (stat.) $\mathrm{MeV}$ is consistent with the nominal value [9]. The measured width of $9.4 \pm 1.5$ (stat.) $\mathrm{MeV}$ is in agreement with the value obtained from the detector simulation.

\section{Limit Calculation}

No signal of a new baryonic state is observed above the $\Xi(1530)^{0}$ mass in either the $X^{0}$ or the $X^{--}$mass spectra. The resonance search can also be performed relative to the observed signal of the known $\Xi(1530)^{0}$ baryon, using the ratio $R$, which is defined as

$$
R(M)=\frac{N^{r e s}(M, q)}{N(1530,0)} \times \frac{\epsilon(1530,0)}{\epsilon(M, q)},
$$

where $N(1530,0) \quad$ represents the number of observed $\Xi(1530)^{0} \rightarrow$ $\Xi^{-} \pi^{+}$and their antiparticle decays. $N^{r e s}(M, q)$, which describes the estimated number of resonance decays depending on the mass $M$ and the charge $q$ of the final state, is derived from the difference between the observed spectra and the expected background contribution. The mass distribution of the hypothetical signal is assumed to be a gaussian with mean $M$ and massdependent width $\sigma(M)$ (obtained from Monte
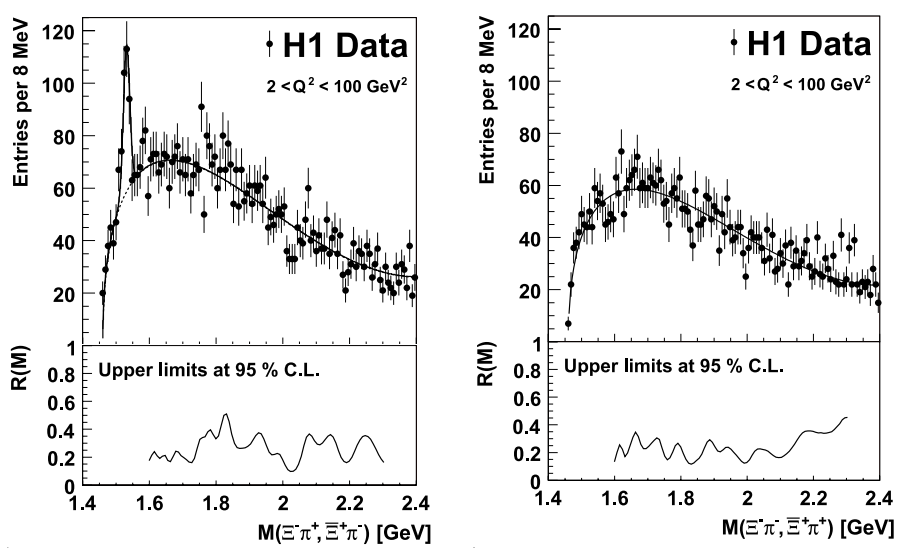

Figure 2: The invariant mass spectrum for the neutral and doubly charged combinations (upper part). The solid line shows the result of a fit to the data using the function defined in the text. The lower part shows the $95 \%$ C.L. upper limit on the ratio $R(M)$ as a function of the mass $M$, as defined in equation 4 .

Carlo simulation) corresponding to the experi-

mental mass resolution. The ratio of efficiencies in equation 4 compensates for the small difference in the reconstruction efficiencies of the $\Xi(1530)^{0}$ baryon and the hypothetical baryon state.

In the ratio $R(M)$ most systematic effects will cancel. This makes it insensitive to detector effects and thus provides a robust quantity for setting upper limits on the production of new narrow baryonic resonances decaying to $\Xi^{-} \pi^{ \pm}$in the mass range $1600-2300 \mathrm{MeV}$. A mass-dependent upper limit at the $95 \%$ confidence level (C.L.) on the ratio $R(M)$ is obtained from the observed invariant mass spectra using a modified frequentist approach based on likelihood ratios [11] analogous to the one applied in [6]. The method takes into account the statistical and systematic uncertainties in the number of signal events and background combinations. 
The final results of the limit calculations are quoted for the kinematic region $2<Q^{2}<$ $100 \mathrm{GeV}^{2}$ and $0.05<y<0.7$, for $p_{\mathrm{T}}(\Xi \pi)>1 \mathrm{GeV}$ and $|\eta(\Xi \pi)|<1.5$. It is assumed that new resonances are produced by a mechanism similar to that of the known $J=3 / 2$ baryons, that they decay into $\Xi \pi$ with a $100 \%$ branching ratio, and that their natural widths are below the experimental resolution.

In the lower part of figure 2 the $95 \%$ C.L. upper limit on the ratio $R(M)$ is presented as a function of the mass $M$. The non-observation of a resonance state in the mass range $1600-2300 \mathrm{MeV}$ limits the production rate of a hypothetical $\Xi_{5 q}^{--}$pentaquark to $12-45 \%$ of the $\Xi(1530)^{0}$ production rate at the $95 \%$ C.L., depending on the $(\Xi \pi)$-mass.

Furthermore, no signal is observed in the neutral invariant mass spectrum in the mass range $1600-2300 \mathrm{MeV}$, above the $\Xi(1530)^{0}$ baryon, limiting the production rate of a hypothetical $\Xi_{5 q}^{0}$ pentaquark state to less than $10-50 \%$ of that of the $\Xi(1530)^{0}$ baryon, depending on the $(\Xi \pi)$-mass.

\section{Conclusions}

A search for new narrow baryonic resonances decaying into $\Xi^{-} \pi^{-}$and $\Xi^{-} \pi^{+}$and their charge conjugate states is performed with the $\mathrm{H} 1$ detector using a DIS data sample. While there is a clear signal from the established $\Xi(1530)^{0}$ baryon, there are no indications of any new baryonic state decaying into $\Xi \pi$ in the mass range $1600-2300 \mathrm{MeV}$. Thus H1 can not confirm the signal reported by the NA49 collaboration. Mass-dependent upper limits at the $95 \%$ C.L. are set on the production ratio of hypothetical states, such as $\Xi_{5 q}^{--}$and $\Xi_{5 q}^{0}$, to the total number of observed $\Xi(1530)^{0}$ baryons. These limits are comparable to those measured by the ZEUS Collaboration [12].

\section{References}

[1] Slides: http://indico.cern. ch/contributionDisplay py? contribId=230\&sessionId=6\& conf Id=9499

[2] D. Diakonov, V. Petrov and M. V. Polyakov, Z. Phys. A 359 (1997) 305.

[3] R. L. Jaffe and F. Wilczek, Phys. Rev. Lett. 91 (2003) 232003 for a review on pentaquark phenomenology see R. L. Jaffe, Phys. Rept. 409 (2005) 1.

[4] M. Karliner and H. J. Lipkin, Phys. Lett. B 575 (2003) 249.

[5] For a review on the experimental search for pentaquark states, see K. H. Hicks, Prog. Part. Nucl. Phys. 55 (2005) 647.

[6] A. Aktas et al. [H1 Collaboration], Phys. Lett. B 639 (2006) 202.

[7] C. Alt et al. [NA49 Collaboration], Phys. Rev. Lett. 92 (2004) 042003.

[8] M. I. Adamovich et al. [WA89 Collaboration], Phys. Rev. C 70 (2004) 022201.

[9] W. M. Yao et al. [Particle Data Group], J. Phys. G 33, 1 (2006).

[10] R. Luchsinger and C. Grab, Comput. Phys. Commun. 76 (1993) 263.

[11] T. Junk, Nucl. Instrum. Meth. A 434 (1999) 435.

[12] S. Chekanov et al. [ZEUS Collaboration], Phys. Lett. B 610 (2005) 212. 